Journal Pre-proof

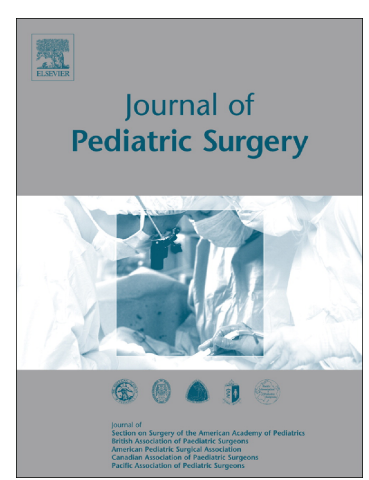

Silvia Ceccanti, Alice Cervellone, Maria Vittoria Pesce, Denis A Cozzi

FEASIBILITY, SAFETY AND OUTCOME OF INGUINAL HERNIA REPAIR UNDER SPINAL VERSUS GENERAL ANESTHESIA IN PRETERM AND TERM INFANTS

PII: $\quad$ S0022-3468(20)30713-2

DOI: $\quad$ https://doi.org/10.1016/j.jpedsurg.2020.09.064

Reference: $\quad$ YJPSU 59957

To appear in: $\quad$ Journal of Pediatric Surgery

Received date: $\quad 15$ May 2020

Revised date: $\quad 22$ September 2020

Accepted date: $\quad 24$ September 2020

Please cite this article as: S. Ceccanti, A. Cervellone, M.V. Pesce, et al., FEASIBILITY, SAFETY AND OUTCOME OF INGUINAL HERNIA REPAIR UNDER SPINAL VERSUS GENERAL ANESTHESIA IN PRETERM AND TERM INFANTS, Journal of Pediatric Surgery (2020), https://doi.org/10.1016/j.jpedsurg.2020.09.064

This is a PDF file of an article that has undergone enhancements after acceptance, such as the addition of a cover page and metadata, and formatting for readability, but it is not yet the definitive version of record. This version will undergo additional copyediting, typesetting and review before it is published in its final form, but we are providing this version to give early visibility of the article. Please note that, during the production process, errors may be discovered which could affect the content, and all legal disclaimers that apply to the journal pertain.

(C) 2020 Published by Elsevier. 


\section{FEASIBILITY, SAFETY AND OUTCOME OF INGUINAL HERNIA REPAIR UNDER SPINAL VERSUS GENERAL ANESTHESIA IN PRETERM AND TERM INFANTS.}

Silvia Ceccanti, $\mathrm{MD}, \mathrm{PhD}^{1}$, Alice Cervellone, $\mathrm{MD}^{1}$, Maria Vittoria Pesce, $\mathrm{MD}^{2}$, Denis A Cozzi, $\mathrm{MD}^{1}$

${ }^{1}$ Paediatric Surgery Unit and ${ }^{2}$ Department of Anesthesia and Critical Care Medicine, Sapienza University of Rome, Azienda Policlinico Umberto I, Rome, RM 00161, Italy

\section{Corresponding author:}

D.A. Cozzi, MD,

Associate Professor of Pediatric Surgery,

Pediatric Surgery Unit,

Sapienza University of Rome,

Viale Regina Elena, 324

00161 Rome, Italy

Telephone and Fax: +39 0649979349

E-mail: da.cozzi@uniroma1.it

\section{Declarations of interest: none}




\section{ABSTRACT}

Background: Inguinal hernia repair (IHR) is a common operation in preterm and term infants. Recently, spinal anaesthesia (SA) has been proposed as an alternative to avoid exposure to general anaesthesia (GA) during early life. The aim of this study was to compare surgical outcomes of open IHR performed under SA versus GA in neonates and infants, and to detect criteria to predict the success or failure of SA.

Materials and methods: This is a 6-year, single centre, non-randomized interventional study (2013-2019). SA was performed with $0.5 \%$ bupivacaine. GA was given using propofol, fentanyl, sevoflurane, and laryngeal mask. Patient demographics, operative time, intraoperative events related to surgery or anaesthesia, and complications were analysed at short and long-term follow-up.

Results: 68 infants (78 IHR) and 37 infants (44 IHR) received SA and GA at he d scretion of the anaesthesiologist, respectively. SA failure rate was $9 \%$, and positively correlated with weigl t at $\mathrm{s}$ irgery $\left(\mathrm{p}=0.001 ; \mathrm{r}_{\mathrm{p}}=0.38\right)$. Conversion from SA to GA occurred in $4(6 \%)$ patients due to prolonged operative tir - $40.75 \pm 4.8$ vs $23.02 \pm 11.3$ minutes; $\mathrm{p}=0.0006)$. There were no differences regarding operative time, intra- and post-operat ${ }^{i} v c$ omplications among the two groups at mean follow-up of $18.53 \pm 21.9$ months.

Conclusions: This pilot study confirms that SA is saf,$t$ feci $^{\prime} \mathrm{e}$ and not detrimental to surgical outcome of neonates and infants undergoing IHR. Additionally, it may help furth. define what patients may have a successful SA. Our experience suggests that SA is especially suitable in infants - - eig. ing $<4000 \mathrm{~g}$, and conversion to GA correlates with prolonged operative time.

LEVEL OF EVIDENCE: I `vel II

KEY WORDS: Inguinal hernia repair, premature, infants, neonate, spinal anaesthesia, general anaesthesia. 


\section{INRODUCTION}

Inguinal hernia is one of the most common conditions requiring early surgery in neonates and preterm infants. Prematurity, bronchopulmonary dysplasia, and increased intra-abdominal pressure resulting from chronic lung disease or gastrointestinal dysfunction may be predisposing factors to the development of inguinal hernia $[1,2,3]$. Surgical repair is conventionally performed under general anesthesia (GA), which may be complicated in approximately $30 \%$ to $35 \%$ of cases by one apneic episode within the postoperative period, especially in otherwise healthy preterm babies [4,5,6]. Additionally, there is concern on the possibility of long-term deleterious effects of anesthesia exposure on the developing brain structure, especially cognitive function and behavioural outcomes after early exposure to GA $[7,8]$.

Some of the larger risks were age $<6$ months, GA duration $>2-3$ hours, and muli, le GA exposures.

Recently, awake regional anesthesia (RA) approaches, including caudal or sp nal b ock, have been proposed as safe and efficient alternative anesthesia method in this frail group of patients $[9,10$. Ho /ever, these techniques continue to be highly underutilized for IHR in neonates and infants probably because, fror su sical point of view, there are still concerns on single dose regional block, which may not be able to ensure a fully ?a . 1late analgesia for the total length of surgery. Additionally, there are also concerns on potential sac disrupti in or jowel herniation due to increased intra-abdominal pressure in awake neonates and infants.

The aim of the present study is to investigate the safety, . Ifectiveness, intra- and post-operative surgical outcome of inguinal hernia repair (IHR) performed under spinal ane sthesı $^{\text {( }}$ SA) only compared to a control group of patients who underwent IHR under GA. Additionally, we made an $a^{+} \_n p_{\imath} \_$develop practical selection criteria for SA in neonates and infants undergoing IHR.

\section{MATERIALS AND ME'HC DS}

We performed a prospective interv ntional non-randomized study of all neonates and infants who underwent elective IHR at our Institution between January 2013 and December 2019. Patients who had received SA or GA were selected, recruited, and followed up. Surgery was considered when patients were hemodynamically stable and spontaneously breathing.

One senior surgeon performed or assisted all IHR, using an open inguinal approach without opening the inguinal canal. The hernial sac was gently peeled off the spermatic cord structures as high as possible, ligated and severed at the level of the internal inguinal ring. All patients followed pre-operative fasting thus allowing an eventual conversion from SA to GA. Two senior anesthetists performed or assisted all the anesthetic procedures. The type of anesthetic technique was chosen at the discretion of the senior anesthetists. Additionally, our protocol included conversion to GA after 3 failed attempts at SA. Bilateral IHR was not considered a contraindication to SA, although the onset of agitation or movements during prolonged operative time were considered indications for conversion to GA. 


\subsection{Spinal anesthesia}

The procedure was performed under sterile conditions with the baby in the sitting position and nurse assisting with positioning to accentuate lumbar kyphosis. Topical anesthetic cream (EMLA) was optional. A sucrose-sweetened pacifier was maintained in the baby's mouth. Since in newborns and infants the spinal cord most frequently terminates at the level of L2/L3, we accessed L4-L5 intrathecal space using a $16 \mathrm{~mm}, 25$-gauge spinal needle, to avoid spinal injury. Access to the intrathecal space was considered successful when cerebrospinal fluid is returned. Local anesthetic used in our patients was $0.5 \%$ isobaric bupivacaine $(1 \mathrm{mg} / \mathrm{Kg})$. Bloody tap was not considered a contraindication for further attempt to SA. Effective motor and sensory block to stimuli was ascertained by gentle pinching of the thigh.

\subsection{General anesthesia}

All patients were induced with sevoflurane in oxygen-air at $7 \mathrm{~L} / \mathrm{min}$ flow via acen ask. Fentanyl $2 \mathrm{mcg} / \mathrm{kg}$ was given intravenously. Induction was done in slow incremental manner up to 5 vo \%. P opofol $2 \mathrm{mg} / \mathrm{kg}$ was given intravenously. Ventilation was controlled by positive pressure mask ventilation afte $\cdots n\llcorner$ occurred, as demonstrated by capnography and respiratory pattern. Laryngeal mask was then positioned. Tracheal intu "ation was performed in case of difficult face mask ventilation, which was defined as unsatisfactory chest moven er ss nd inability to oxygenate the patient $(\mathrm{SpO} 2<90 \%$ with $\mathrm{FiO}_{2}=1,0$ ), secondary to either functional or anatom; al aus. No muscle relaxants were used.

All patients had an IV placement, regardless of the type ${ }^{f}$ anesthesia received. IV was placed before performing SA and after mask induction in case of GA.

No other block was performed to provide $\mathrm{pr}\lrcorner$ n $p_{\ldots}$. tive analgesia. Oral or intravenous paracetamol were prescribed pro re nata (PRN) for postoperative pain, whic! was assessed using a well-validated 10-point FLACC score (Face, Legs, Activity, Cry, Consolability) [11]. Paracetamc' was siven if FLACC score $\geq 4$ (i.e. pain intensity scores in the moderate to severe range). All preterm or $<3$ mrnth. old lfants were kept overnight.

\subsection{Outcome measured}

Demographics, gestational age, age at surgery, time from placement of the spinal anesthetic to end of operation, success of spinal puncture, success of attaining adequate surgical anesthesia, need for supplemental systemic sedation, and conversion to GA, were prospectively recorded in an electronic database. Measured intra-operative complications included relevant bleeding, bowel injury or rupture of the hernial sac. Additionally, early post-operative complications such as wound infection and hematoma were also recorded. Median arterial pressure (MAP) was measured at skin incision and 15 minutes after beginning of surgery. Any hypotension was defined as MAP of $<45 \mathrm{mmHg}$. Apnea episodes were monitored until discharge. Apnea was defined as a pause in breathing $>15$ seconds or $>10$ seconds if associated with $\mathrm{O}_{2}$ saturation $<80 \%$ or bradycardia (20\% fall in heart rate). 
We routinely offered clinical assessment at 1 and 6 months' post-operative follow-up. Additionally, we made an attempt to invite patients to a special clinic follow-up, to assess the following surgical outcome measures: testicular size and position, and/or inguinal hernia recurrence, and/or metachronous inguinal hernia.

\subsection{Statistical analysis}

Continuous variables were expressed as mean (SD). Differences between the two groups were evaluated using two-tailed unpaired t test and Fisher's exact test, as appropriate. Pearson's correlation coefficient was used to evaluate relationship between variables potentially related to failure or conversion of SA. $\mathrm{p}<0.05$ was regarded as significant. Statistical analysis was implemented with SPSS 13.0 statistical software (IBM SPSS Statistics for Windows, Version 22.0. IBM Corp, Armonk, New York, United States). Ethical approval was obtained for this study rom our institutional review board (dip.ped.npi: odg $\mathrm{n}^{\circ}$ 9/ 29.01.2013) and informed consent was obtained from 11 pa ents.

\section{RESULTS}

During the study period, we performed 105 surgical procedures for $\mathrm{\imath} \iota$ al of 122 open IHR in preterm or term infants. Table 1 summarizes patient demographics and characteristics. Post ' $\mathrm{O}^{\prime} \mathrm{c} \in \mathrm{s}$ tional age at surgery was $41.27 \pm 3.43$ and $45.07 \pm 6.0$ days for patients undergoing SA and GA, respectivel (p $: 0 . \iota^{\urcorner} 91 ; 95 \% \mathrm{CI}$ : 1.977 to 5.623 ). Seventy-two patients were electively admitted from home, while the remaining 33 、 sre hospitalized in NICU, without differences between the two groups regarding the associated medical problem - as escribed in table 1.

First-line SA was attempted in 75 infants. $H_{\text {, . }}$, $_{-}$, the initial SA attempt failed (cerebrospinal fluid was not obtained) in 7 (9\%) infants weighing between $3790 \mathrm{~g}$ ar $163 \mathrm{\partial} \mathrm{g}$, who were eventually included in the GA group. These patients had a

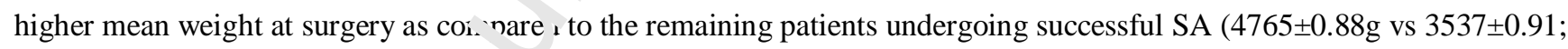
$\mathrm{p}=0.001 ; 95 \% \mathrm{CI}:-1948.3$ to -50 62). There was a positive correlation between higher weight and failure of $\mathrm{SA}$ ( $\mathrm{p}=0.001$; $\left.r_{p}=0.38\right)$. Overall, 68 infants finall underwent IHR under SA, including 10 with bilateral inguinal hernia, whereas the remaining 37 infants, 7 with bilateral inguinal hernia, underwent IHR under GA. Three patients of SA group had a concomitant undescended testis, and successfully underwent orchiopexy during the same procedure. There was no difference in laterality of inguinal hernia, mean gestational age and concomitant respiratory, cerebral and cardiac associated problems between the two groups (Table 1).

SA was successful for IHR completed within $29.34 \pm 10.47$ minutes from initial spinal puncture. We found a positive correlation between prolonged operative time and rate of conversion to $\mathrm{GA}\left(\mathrm{p}=0.001 ; \mathrm{r}_{\mathrm{p}}=0.41\right)$. Conversion from SA to GA occurred in $4(6 \%)$ patients because of the onset of agitation and movements during a prolonged operative procedure $(43.75 \pm 4.78$ vs $23.02 \pm 11.26$ minutes, $\mathrm{p}=0.0006 ; 95 \% \mathrm{CI}:-32.12$ to -9.35$)$. This prolonged duration of surgery was due to 
complicated bilateral IHR $(n=2)$, troublesome repair of Amyand hernia adherent to the sac $(n=1)$, and because of a significant time gap between skin incision and initial spinal puncture $(n=1)$.

One $(2.7 \%)$ patient undergoing GA experienced bradycardia after anesthetic induction with sevoflurane, which was rapidly reversible after positive pressure ventilation. Additionally, 1 (2.7\%) ex-preterm patient developed a laryngospasm at the insertion of laryngeal mask, which required tracheal intubation and intensive care unit observation, thus prolonging his hospital stay. No patient experienced hypotension in either group. However, the MAP measured at skin incision was significantly lower in the GA group $(49.94 \pm 12.2$ vs $62.72 \pm 16.8 ; \mathrm{p}=0.009 ; 95 \% \mathrm{CI}$ : -22.183 to -3.377$)$, but such difference was no longer significant at 15 minutes after the beginning of surgery $(55.83 \pm 14.6 v s .48 .83 \pm 12.8 ; \mathrm{p}=0.11 ; 95 \% \mathrm{CI}$ : -15.737 to 1.737$)$. No patient in the SA group developed urinary retention.

All patients received full formula soon after the end of surgical procedure. Th re $\mathrm{w}$ is no difference within the two groups regarding intra and post-operative complications. There was no bowel inj, ry no $r$ wound complications in both study group. We found no significant difference in the use of postoperative analg ${ }^{\prime} a_{\S}$ nts between the two groups. One ex-preterm infant (born at 26 weeks of gestation and operated at 12 weeks of are) . cluded in the GA group, experienced an episode of early post-operative apnea during the first 30 minutes after th $\cdot f . l d$ of surgery. No patient in the SA group experienced postoperative apnea. Home discharge was possible withir $\angle 4$ iou. for all patients electively admitted ( $\mathrm{n}=72)$, except one who required prolonged hospital stay because of a laryngospa $m$ during GA. At a median overall post-operative follow-up of 8 months (range 1 to 78), 4 patients of SA group a $r^{2}$ ?tients of the GA group presented a metachronous inguinal hernia. One patient of the GA group developed ingr... ${ }^{1} l_{\ldots} \ldots$ nia recurrence 2 weeks after surgery. Finally, 2 patients of the SA group developed testicular atrophy. Of these, 1 ratien. underwent reduction of incarcerated inguinal hernia before IHR, and the other patient had an associated ipsila aral ı ıdescended testis, operated at the same time of IHR.

\section{DISCUSSION}

Prematurity is the single most important predisposing factor for the development of inguinal hernia [1]. The incidence increases with decreasing birth weight [2], counting around 38\% of infants with a birth weight between $751 \mathrm{~g}$ and $1000 \mathrm{~g}$ [12], and around $16 \%$ of infants with a birth weight between $1001 \mathrm{~g}$ and $1250 \mathrm{~g}$ [2,13]. Given the high probability of preoperative incarceration of the inguinal hernia in neonates and infants, occurring in up to $60 \%$ of cases within the first 6 months of life [14], prompt treatment immediately after diagnosis is strongly recommended.

Moreover, several studies demonstrated that about $30 \%$ to $35 \%$ of otherwise healthy ex-preterm infants undergoing IHR under GA at a post conceptual age less than 45 weeks, will experience one or more episodes of apnea in the postoperative period $[4,5,6]$. This incidence decreases to $5.9 \%$ in patients $45-60$ weeks post conceptual age. Even though our SA group patients had a significantly lower post conceptual age, none of them developed post-operative apnea. In contrast, the single 
episode of post-operative apnea in our series occurred in 1 (2.7\%) patient in the GA group. Such incidence was similar to that reported by Davidson et al. [15], who found that the incidence of early apnea (0 to 30 minutes) was higher in the GA group respect to patients undergoing any type of regional anesthesia (RA) (3\% vs. $1 \%$; $\mathrm{p}=0.03)$. However, in their study, the incidence of late apnea (30 min - 12 hours) was no different between the two groups.

Therefore, the avoidance of post-operative apnea may be a significant advantage of SA over GA, thus potentially allowing earlier home discharge, regardless of the post conceptional age.

Additionally, there is still a concern and debate on GA as potential risk factor for impaired neurodevelopmental outcome in preterm babies and neonates [7,8]. Conversely, a recent multicenter randomized trial comparing GA vs awake-RA in infants (including either SA, caudal anesthetic, or a combined spinal-caudal anesthetic) emonstrated that there is no or very minimal risk of adverse developmental consequences in infants undergoing $\mathrm{o}_{\perp}$ eratı ns under GA lasting less than 2 hours, such as IHR [16].

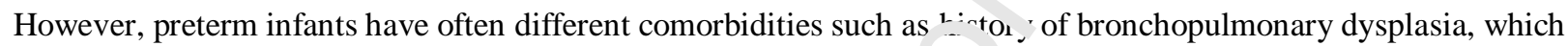
increases the risk of respiratory complications during and after GA. $\mathbf{1}_{\mathbf{L}} \cdot$ refore, the use of SA may be a considerable advantage in this frail group of preterm or ex-preterm patient. $r_{,} \mathrm{V} v \mathrm{ig}$ a more qualitative measure of recovery characteristics.

Additionally, home discharge was possible within 24 ho "s for all patients electively admitted, but 1 who required prolonged hospital stay because of a laryngosparn du. ing GA. In our experience, SA was feasible and successful in $91 \%$ of attempts. Failure in the spinal needle placer _..+ n... probably due to the greater body weight at operation of a subset of patients, especially at risk if weighing $\geq 000_{\mathrm{s}}$. We hypothesized that the higher is the weight, the more difficult is to access the intrathecal space using a $: 5 \mathrm{~mm}$ needle, as that used in our cohort of patients. Moreover, the bigger the baby, the harder is to hold him and avcid it mor ements during spinal needle placement, thus making SA more difficult. As far as concerns the sitting position durins SA for IHR, this was our anesthetists' preference, merely because they were most familiar with. Notably, both lateral and sitting positions have been successfully used for SA in neonatal and infantile IHR [9], but no outcome comparison between the two positions has been conducted in infants to date.

Additionally, SA failure occurred homogeneously throughout the study period, and independently of whether performed by senior anesthetist or trainee, thus suggesting no correlation with learning curve, although the numbers are too small to extrapolate any firm conclusion.

Frawley $\mathrm{G}$ et al. reported that the primary reason for failure of any type of RA was a bloody tap [9], which has been reported as high as $46 \%$ in previous studies [10]. In our experience, bloody tap was not considered a contraindication for further attempt to SA, which was successfully performed at the second attempt. Notably, none of our patients requiring conversion to GA presented a bloody tap. 
Although spinal complications following SA are rare, we believe that our policy to puncture the L4-L5 intrathecal space may be a safe and effective measure to prevent potential spinal cord injury. Our technique is similar to that already reported by others [9]. Notably, a recently published anatomical study has showed that the conus medullaris in newborns was not found caudal to the L3 vertebral body [17].

An important limitation to SA is that it provides a relatively short duration of surgical anesthesia, generally thought to be less than $60 \mathrm{~min}$ for a single spinal block anesthetic injection. Longer time extension may be obtained up to 110 minutes if epinephrine or clonidine is added to the local anesthetic $[18,19]$. We routinely avoid the addition of epinephrine because our operative time for open IHR, performed without opening the inguinal canal, is usually $<30$ minutes, as we believe in many other pediatric surgery centers.

In our experience, single dose SA was enough to obtain analgesia for the durc ion i $f$ the procedure without the need for additional sedation in $94 \%$ of cases. The $6 \%$ conversion rate from SA to ( A in sur series was probably due to the prolonged operative time after induction of SA, thus demonstrating $t^{2} \ldots+$ thi $>$ type of anesthesia is especially suitable for procedures lasting up to 40 minutes from spinal puncture, regardles $\omega_{1}$ 'ne unilateral or bilateral repair. However, complicated inguinal hernia may require longer operative tim , 'nu ; resulting in the likelihood of conversion to GA, as occurred in 1 patient of our series who had an Amyar $\lrcorner \mathrm{n}$ rnla dherent to the sac. Therefore, in case of prolonged operative times for any reason, the anesthetist should be sady to convert the procedure into GA or to give a brief supplementation of the SA by mask GA.

To date, the exclusively awake regional ane $\therefore$ ati $\ldots$ pproach for IHR has not obtained widespread consensus among pediatric surgeons because of the fear th ? wilt.out GA, the child may cry and/or present movements increasing intraabdominal pressure, which may lead ` $`$ int stinal herniation during the procedure and/or rupture of the hernial sac. In our experience, these issues $\mathrm{rid} \mathrm{r} \mathrm{t}$ aft :ct either the operative time nor the post-operative outcome, including the risk of hernia recurrence or testicular dam ige. In case of intra-operative agitation, crying, or movements of the upper part of the body, gentle manual containment and/or a sucrose-sweetened pacifier maintained in the baby's mouth were successful to obtain control of the baby, instead of switching to GA.

Our outcomes on SA parallel those reported in a previous Cochrane review and in a subsequent randomized study, which did not demonstrate any difference between all types of RA and GA in preterm infants undergoing IHR, in terms of duration of surgery and average length of hospital stay [20,21]. Regarding postoperative pain, we adopted the FLACC score, which is a validated behavioral scale for scoring postoperative pain in young children who are unable to communicate their pain [11]. Notably, a recent randomized study found that FLACC scores were significantly lower in the RA vs the GA group [21]. In our experience, we used simply paracetamol for post-operative pain relief, and we did not find any significant difference between infants receiving SA as compared to those receiving GA. Notably, we do not 
use routinely any wound infiltration or caudal analgesia after IHR, and generally paracetamol is prescribed PRN, being required very rarely also in older children. The less postoperative analgesic requirement in our patients may be presumably due to the adopted IHR technique, which does not include opening of the inguinal canal. Furthermore, in our series we did not experience potential adverse effects described also during RA such as hypotension, bradycardia, or respiratory failure. In contrast, 3 patients in the GA group experienced intraoperative bradycardia $(n=1)$, laryngospasm requiring mechanical ventilation support for 2 days $(n=1)$, and post-operative apnea $(\mathrm{n}=1)$.

Of greater importance and impact may be the better hemodynamic stability and preserved cerebral autoregulation of infants during SA vs. GA. McCann et al found that RA reduces the incidence or vpotension and chance of intervention to treat it compared with GA in infants undergoing IHR [22]. Notably, when omp ring different types of RA, hemodynamic stability was better after SA vs caudal anesthesia [23]. In o ' $r$ ser es, there were no significant episodes of hypotension in either group, although GA patients had a lower MAP : $\mathrm{u}_{\text {. }} \cdot$ ented at skin incision.

Recently, Gurria et al. has also reported their successful use of RA in v ry preterm neonates undergoing IHR [24]. Notably, they found that $17 \%$ of their patients undergoing G $t$ e pr rienced prolonged intubation beyond 4 hours after surgery. This was also the case for 1 of our ex-preterr. $\cup \backslash$ pa: ${ }^{\text {ents, }}$ who required prolonged intubation following a laryngospasm.

Although there is a large anesthetic literature on ${ }^{\top} A_{1}$. infants, limited information is available on this important technique from the surgical standpoint. In $0^{\prime} .{ }^{\prime} x_{p} \ldots$ ence, we did not find any difference with regard to surgical outcome between patients who received ' GR u.der GA or SA at a long-term follow-up. Despite the reported fear of rupture of the hernial sac and intestii. 1 hes uiation disturbing the correct surgical procedure during SA, we did not experience a higher risk of $\mathrm{h} r$ rnia recu rence in this group. The two testicular atrophies occurred in a patient with a previous history of incarcerated ip lateral inguinal hernia and in a patient with concomitant undescended testis, respectively.

Our $2.8 \%$ rate of testicular atrophy parallels the higher incidence of testicular atrophy reported in some specific population of infants undergoing IHR. Conditions predisposing to higher risk of testicular atrophy following IHR include previous hernia incarceration (14\%) [25], prematurity (10\%) [26], and ipsilateral undescended testis undergoing concomitant orchiopexy (12\%) [25].

There are a number of limitations in our study. First, we acknowledge the relatively small sample size of our population, although present series is the largest single center experience using only SA among different types of RA for open IHR. Additionally, there were significant differences in birth weight, weight at surgery and age at surgery among the two study groups, presumably related to the anesthetist's choice of SA for more critical babies, who were 
thought to be at higher risk of GA complications. Despite these limitations, our goal with this pilot study was to share our experience, thus contributing to the growing awareness of SA utility and feasibility in infants undergoing IHR.

\section{CONCLUSIONS}

From our experience, it appears that SA is feasible, safe, and effective in neonates and infants undergoing IHR, without affecting surgical intraoperative or long-term outcomes at specialized pediatric surgical centers. This type of anesthesia has proven to be particularly suitable in small patients with prematurity-related comorbidity, when risks of GA should be weighed against the risks of non-intervention. A body weight of $4000 \mathrm{~g}$ and/or expected operative time up to 40 minutes represented our upper limits of predictability for successful SA. Howev, a prospective randomized control trial with an adequate number of patients may be needed to confirm the result : of o ir pilot study. 


\section{REFERENCES}

[1] Grosfeld JL. Current concepts in inguinal hernia in infants and children. World J Surg 1989;13:506-515. doi:10.1007/BF01658863

[2] Kumar VH, Clive J, Rosenkrantz TS, Bourque MD, Hussain N. Inguinal hernia in preterm infants $(<$ or $=32$-week gestation). Pediatr Surg Int 2002;18:147-152. doi:10.1007/s003830100631

[3] Powell TG, Hallows JA, Cooke RW, Pharoah PO. Why do so many small infants develop an inguinal hernia? Arch Dis Child 1986;61:991-995. doi:10.1136/adc.61.10.991

[4] Gollin G, Bell C, Dubose R, et al. Predictors of postoperative respiratory complications in premature infants after inguinal herniorrhaphy. J Pediatr Surg 1993;28:244-247. doi:10.1016/s0022-34（

[5] Sims C, Johnson CM. Postoperative apnoea in infants. Anaesth Intensive are 994;22:40-45. doi:

\section{$10.1177 / 0310057 X 9402200108$}

[6] Massoud M, Kühlmann AYR, van Dijk M et al. Does the Incider .. n1 ? ostoperative Complications After Inguinal Hernia Repair Justify Hospital Admission in Prematurely and Term Bı $\eta$ Infants? Anesth Analg 2019;128:525-532. doi:10.1213/ANE.0000000000003386

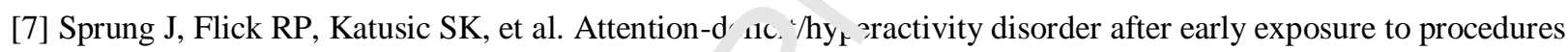
requiring general anesthesia. Mayo Clin Proc 2012; 87:. ?n-129. doi:10.1016/j.mayocp.2011.11.008

[8] Lin EP, Lee JR, Lee CS, Deng M, Loepke Aw/ L、anesthetics harm the developing human brain? An integrative analysis of animal and human studies. Neur $\ldots$ xic . Teratol. 2017;60:117-128. doi:10.1016/j.ntt.2016.10.008

[9] Frawley G, Bell G, Disma N, Withir``on LE et al. Predictors of Failure of Awake Regional Anesthesia for Neonatal Hernia Repair: Data from the Gener.' An s hesia Compared to Spinal Anesthesia Study--Comparing Apnea and Neurodevelopmental Outcom as. . nest lesiology 2015;123:55-65. doi: 10.1097/ALN.0000000000000708.

[10] Pinheiro JM, Furdon S, Ocho: LF. Role of local anesthesia during lumbar puncture in neonates. Pediatrics. 1993; 91:379-382.

[11] Merkel SI, Voepel-Lewis T, Shayevitz JR, Malviya S. The FLACC: a behavioral scale for scoring postoperative pain in young children. Pediatr Nurs 1997;23:293-297.

[12] Peevy KJ, Speed FA, Hoff CJ. Epidemiology of inguinal hernia in preterm neonates. Pediatrics 1986;77:246-247.

[13] Rajput A, Gauderer MW, Hack M. Inguinal hernias in very low birth weight infants: incidence and timing of repair. J Pediatr Surg 1992;27:1322-1324. doi:10.1016/0022-3468(92)90287-h

[14] Boocock GR, Todd PJ. Inguinal hernias are common in preterm infants. Arch Dis Child 1985;60:669-670. doi:10.1136/adc.60.7.669 
[15] Davidson AJ, Morton NS, Arnup SJ, et al. Apnea after Awake Regional and General Anesthesia in Infants: The General Anesthesia Compared to Spinal Anesthesia Study--Comparing Apnea and Neurodevelopmental Outcomes, a Randomized Controlled Trial. Anesthesiology. 2015;123:38-54. doi: 10.1097/ALN.0000000000000709.

[16] Davidson AJ, Disma N, de Graaff JC, et al. Neurodevelopmental outcome at 2 years of age after general anaesthesia and awake-regional anaesthesia in infancy (GAS): an international multicentre, randomised controlled trial. Lancet. 2016;16;387:239-250. doi: 10.1016/S0140-6736(15)00608-X.

[17] Van Schoor AN, Bosman MC, Bosenberg AT. Descriptive study of the differences in the level of the conus medullaris in four different age groups. Clin Anat. 2015;28:638-644. doi:10.1002/ca.22505.

[18] Trifa M, Tumin D, Whitaker EE, Bhalla T, Jayanthi VR, Tobias JD. Spinal nesthesia for surgery longer than 60 min in infants: experience from the first 2 years of a spinal anesthesia progral . J A 1esth. 2018;32:637-640. doi: $10.1007 / \mathrm{s} 00540-018-2517-5$.

[19] Rochette A, Raux O, Troncin R, Dadure C, Verdier R, Capdevi' ${ }^{1}$. . . C'onidine prolongs spinal anesthesia in newborns: a prospective dose-ranging study. Anesth Analg 2004;98:5c -59. doi:10.1213/01.ane.0000093229.17729.6c [20] Jones LJ, Craven PD, Lakkundi A, Foster JP, Badawi N. R -gi nnal (spinal, epidural, caudal) versus general anaesthesia in preterm infants undergoing inguinal he nu rha hy in early infancy. Cochrane Database Syst Rev 2015;9:CD003669. doi:10.1002/14651858.CD003669.p، `2

[21] Disma N, Withington D, McCann ME, et a ${ }^{1} \mathrm{Su}_{8}$ 'cal practice and outcome in 711 neonates and infants undergoing hernia repair in a large multicenter RCT: Se _, ^di., results from the GAS Study. J Pediatr Surg. 2018;53:1643-1650. doi: 10.1016/j.jpedsurg.2018.01.003.

[22] McCann ME, et al. Differences in Blc ud Pressure in Infants After General Anesthesia Compared to Awake Regional Anesthesia (GAS Srıdy A Pı spective Randomized Trial). Anesth Analg 2017;125:837-845. doi:10.1213/ANE.0000000000001;70

[23] Seyedhejazi M, Moghadam A, Sharabiani BA, Golzari SE, Taghizadieh N. Success rates and complications of awake caudal versus spinal block in preterm infants undergoing inguinal hernia. Saudi J Anaesth. 2015;9:348-352. doi:10.4103/1658-354X.154704

[24] Gurria J, Kuo P, Kao A, Christensen L, Holterman A. General endotracheal vs. non-endotracheal regional anesthesia for elective inguinal hernia surgery in very preterm neonates: A single institution experience. J Pediatr Surg 2017;52:56-59. doi:10.1016/j.jpedsurg.2016.10.019

[25] Wright NJ, Davidson JR, Major C et al. The management of boys under 3 months of age with an inguinal hernia and ipsilateral palpable undescended testis. J Pediatr Surg 2017;52:1108-1112. doi.org/10.1016/j.jpedsurg.2017.02.011 
[26] Fette AM, Höllwarth ME. Special aspects of neonatal inguinal hernia and herniotomy. Hernia 2001;5:92-96. doi:10.1007/s100290100011 
Table 1: Patient demographics and characteristics

\begin{tabular}{|c|c|c|c|}
\hline & $\begin{array}{c}\text { Spinal Anesthesia } \\
(n=68)\end{array}$ & $\begin{array}{c}\text { General Anesthesia } \\
\qquad(\mathrm{n}=37)\end{array}$ & p value \\
\hline $\mathrm{M}: \mathrm{F}$ & $61: 7$ & $33: 4$ & 1 \\
\hline Bilateral IHR & 10 & 7 & 0.58 \\
\hline Gestational age (wks) & $33.1 \pm 4.6$ & $34.5 \pm 5.1$ & 0.16 \\
\hline Post conceptional age (wks) & $41.27 \pm 3.43$ & $45.07 \pm 6.0$ & 0.0002 \\
\hline Birth weight (g) & $1178 \pm 1.190$ & $2.309 \pm 969$ & $<0.0001$ \\
\hline Weight at surgery $(g)$ & $2174 \pm 1.820$ & $4709 \pm 1.321$ & 0.002 \\
\hline Age at surgery (mos) & $1.9 \pm 0.9$ & L. . \pm 1.4 & 0.004 \\
\hline \multicolumn{4}{|l|}{ Associated problems } \\
\hline Broncopulmunary dysplasia/RDS & 8 & 6 & 0.55 \\
\hline Intraventricular hemorrhage & 5 & 2 & 1 \\
\hline Brain anomalies & 2 & 1 & 1 \\
\hline Cardiovascular anomalies & 170 & 8 & 0.81 \\
\hline Previously incarcerated inguinal hernia & 2 & 3 & 0.35 \\
\hline Associated undescended testis & 3 & 0 & 0.55 \\
\hline Intra- and post-operative details & & & \\
\hline Operative Time (min) & $24.49 \pm 13.0$ & $28.40 \pm 13.73$ & 0.11 \\
\hline Time from SA puncture to end surgery $\left(n_{i} \eta\right)$ & $29.34 \pm 10.47$ & - & - \\
\hline Inpatients & $24(35 \%)$ & $7(19 \%)$ & 0.11 \\
\hline Intra-operative complications & 0 & 2 & 0.12 \\
\hline Hernia recurrence & 0 & $1(2 \%)$ & 0.39 \\
\hline Testicular atrophy & $2(2.8 \%)^{*}$ & $0(0 \%)$ & 0.53 \\
\hline Post-operative F-U (mos) & $18.33 \pm 54$ & $18.83 \pm 20.65$ & 0.41 \\
\hline
\end{tabular}

RDS: respiratory distress syndrome; ${ }^{\circ} 1$ patient had a previous surgical repair of patent ductus arteriosus;

* Rate calculated on a total of 71 male IHR; Data are mean \pm SD or $n$. 